\section{SP6-30 ASSESSMENT OF COMMUNITY AWARENESS TOWARDS HEALTHY LIFESTYLES IN UAE}

doi:10.1136/jech.2011.142976q.1

M Fikri, S El Badawi. Ministry of Health, Dubai, United Arab Emirates

Introduction A study was carried out in UAE in June 2009 for assessment of community awareness towards healthy lifestyle (HLS). The objective of the study was to; assess the awareness of community pertaining to risk factors associated with NonCommunicable Diseases (NCDs), to identify the source of information of respondent HLS knowledge and to recommend effective interventions for improved awareness.

Methods The study was cross sectional quantitative descriptive. The sample unit was the attendant to Primary healthcare Centers in the northern emirates. The sample size was 2034 local and resident Arabic speaking population aged ranged from 18 to 65 years. 53\% of them were females while the rest were males.70\% were local residents.

Results $74 \%$ of respondent were found to correlate between type of food and NCDs. 57\% thought low carbohydrate and fat diet were suitable for weight loss.16.2\% were found to be currently smoking. One fifth $(23 \%)$ were able to relate smoking to increased risk of diabetes. only $10 \%$ thought that Hubble bubble is less hazardous to health from cigarettes. $95.6 \%$ knew about the positive relation of physical activity (PA) and good health, but only $46 \%$ identified correctly the recommended duration and frequency of PA. Main source of information for respondents for their knowledge was T.V (83\%), newspapers and magazine (69\%) health workers (37\%).

Conclusion The study recommended the use of optimal means to raise the awareness of public in relation to HLS

\section{SP6-31 ROOT CAUSE ANALYSIS FOR PATIENT SAFETY ISSUES IN A PSYCHIATRY UNIT OF A GENERAL HOSPITAL}

doi:10.1136/jech.2011.142976q.2

N Bedi.* Gandhi Medical College, Bhopal, Madhya Pradesh, India

Patient safety issues are important due to patients cognitive declines and physical inactivity in mental institutions. A Root cause analysis is applied to analyse the deaths among psychiatric patients admitted in ward of a teaching hospital with an aim to study various patient safety factors contributing to the deaths for the last 2 years. It is situated a little distance from the main inpatient building of the hospital. Case records of all deaths (four in all) were obtained and root cause analysis was carried out, both from examination of the case records as well as the departmental death review. About 500 patients admitted every year accompanied by a relative and four deaths reported in last 2 years (2008-2009) that is, $0.8 \%$. Three males and one female reported death. The cases were diagnosed as Bipolar Disorder current episode mania, Alcohol dependence, Grief/ Psychosis, Post head injury psychosis with causes of deaths were aspiration pneumonitis, alcohol poisoning, aspiration and irreversible shock with hypothermia. The Root cause analysis of organisational/management, work environmental factors, team/staff, patients factors, task factors revealed the specific and general contributory factors were distance of the ward from the rest of the hospital, poor communication among staff and responses of the hospital emergency teams to SOS from Psychiatry and poor recognition of serious physical factors/symptoms. A planned future intervention patient safety was recommended suggesting the availability of heater and blankets, timely transfer, implementation of SOPs for patients work up immediately after admission and proper team coordination for patients referral.

\section{SP6-32 MONITORING OF TB-HIV CO-INFECTION CASES DISCHARGED FROM A REFERRAL HOSPITAL IN SAO PAULO, BRAZIL}

doi:10.1136/jech.2011.142976q.3

1,2V Souza-Pinto, ${ }^{* 2}$ R Bammann. 'Sao Paulo State's Secretary of Health, TB Control Program, Sao Paulo, Sao Paulo, Brazil; ${ }^{2}$ Sao Paulo State's Secretary of Health, Emilio Ribas Institute for Infectious Diseases, Sao Paulo, Sao Paulo, Brazil

Referral public services are essential concerning TB-HIV co-infected patients, but they do not exempt from the geographical and social complicators where they are inserted in. Retrospective data analysis of the TB Surveillance and Information System (TBWEB) regarding co-infected TB/HIV inpatients discharged from July 2008 to June 2009. Were included 209 consecutive cases. From those, 47 (22.5\%) were not notified to TBWEB. From the 162 (77.5\%) notified cases under TB treatment initiated or sustained while in hospital, $60(37.0 \%)$ presented pulmonary-TB, other 60 (37.0\%) extrapulmonary-TB and $42(26.0 \%)$ mixed forms. 99 cases (61.1\%) were considered as a new TB case, 29 (17.9\%) were re-treatments after default, $5(3.1 \%)$ were re-treatments after failure and $29(17.9 \%)$ had been relapsed. There was no culture record in 60 (37.0\%) cases. In other 102 , culture was positive in $68(42.0 \%)$. Sensitivity test was performed in 54 of these $68(79.4 \%), 37$ stains were all-sensitive (68.5\%), 5 (9.3\%) were monoresistant, and $12(22.2 \%)$ were multiresistant to TB-drugs. Cure was achieved in $45.7 \%$ of the 162 notified cases; default in $29.6 \%$; failure in $3.7 \%$; change of diagnosis in $3.7 \%$ (due to non-tuberculosis mycobacteria); $3.7 \%$ remain under treatment. Were notified 22 deaths (13.6\%). The high rates of underreporting, cultures and sensitivity tests not performed and, most of all, the low rate of cure urges practical and effective measures to improve this reality. A deeper analysis of these data are mandatory for better understanding and searching for solutions. A stronger commitment to DOTS Strategy rises as a possible answer.

\section{SP6-33 ESTIMATION OF THE CONTRIBUTION OF ASSISTED AND NON-ASSISTED REPRODUCTIVE TECHNOLOGY FERTILITY TREATMENTS TO MULTIPLE BIRTHS DURING THE LAST 30 YEARS IN JAPAN: 1977-2008}

doi:10.1136/jech.2011.142976q.4

S Ooki. ${ }^{*}$ Ishikawa Prefectural Nursing University, Kahoku, Ishikawa, Japan

Introduction The purpose of the present study was to estimate the effect of fertility treatment; both assisted reproductive technology (ART) and non-ART ovulation stimulation, on the number and rate of multiple live births during the last 30 years in Japan.

Methods Japanese vital statistics according to maternal age class and plurality of live births published by the Ministry of Health, Labour and Welfare from 1974 to 2009 and Japanese ART statistics published by Japan Society of Obstetrics and Gynaecology from 1989 to 2008 were gathered and reanalysed. Total iatrogenic multiple births were estimated by vital statistics assuming that spontaneous multiple-birth rates according to maternal age class would be constant. The number and rates of ART between 1977 and 1988 were estimated using approximation formulae. The number of non-ART iatrogenic multiple births were estimated by subtracting ART multiples from total iatrogenic multiples.

Results The birth rates of iatrogenic multiples increased dramatically between 1977 and 2005, and decreased. The estimated maximum percentage of multiple births that were iatrogenic was $50.0 \%$ in 2005 . Non-ART multiple births increased during past 30 years overall, whereas ART multiples tended to increase from 1983 to 2005 and then decreased rapidly. The number or percentage of ART multiples were almost constantly lower than that of non-ART multiples. 